GAM FOR THE HCSI TEST

\title{
Improving Mouse Behaviour Assessment with Generalized Additive Modelling (GAM): The Case of the Home Cage Social Interaction Test
}

\author{
Giovanni Sala ${ }^{1} \&$ Bruno Galuzzi ${ }^{2}$
}

${ }^{1}$ Fujita Health University, Institute for Comprehensive Medical Science (ICMS)

${ }^{2}$ University of Milano-Bicocca, Department of Computer Science, Systems and Communications

Corresponding author: Giovanni Sala, Institute for Comprehensive Medical Science (ICMS), Fujita Health University, 1-98 Dengakugakubo Kutsukake-cho, Toyoake, 470-1192, Japan. Email: sala.giovanni475@gmail.com

\section{Acknowledgements}

We gratefully thank Satoko Hattori for her help with the Mouse Phenotype Database and Fernand Gobet for revising an earlier draft of this manuscript. This work was supported by the Japan Society for the Promotion of Science [20K14267 granted to GS].

\section{Data Accessibility Statement}

Data and code are available at https://osf.io/j7uyt/. 


\title{
GAM FOR THE HCSI TEST
}

\begin{abstract}
The Home Cage Social Interaction (HCSI) test is a standard tool for assessing locomotor activity and social behaviour in numerous mouse models of psychiatric disorders. Data obtained from this test are commonly analysed with linear modelling. However, linear modelling is unfit for HCSI data because the relation between HCSI response variables and time is cyclic rather than linear. Moreover, the response variables are commonly assumed to follow a Gaussian (normal) distribution, which is pretty much never the case with HCSI data. In brief, the statistical model currently applied to HCSI data is substantially incorrect.

We thus propose to employ nonlinear modelling techniques such as General Additive Modelling (GAM) for HCSI data. GAM utilizes smoothing functions that allow for nonlinear relationships between the response variable and the covariates. Moreover, GAM enables the researcher to employ smoothing functions designed for analysing cyclic data. Finally, GAM also implements nonGaussian exponential family distributions to meet the model's statistical assumptions.

We compare linear modelling and GAM on 27 HCSI statistically independent experiments involving wild-type and mutant mice over seven days. In all the cases, the GAM models outperform the linear models in both explained deviance and inference reliability. Most notably, the linear models tend towards Type I error with regard to the group effect and Type II error with respect to the time-by-group interaction. We thus recommend researchers to adopt the present statistical model to analyse HCSI data.
\end{abstract}

Keywords: generalized additive modelling; home cage interaction test; mouse behaviour; nonlinear modelling; psychiatric disorders. 
GAM FOR THE HCSI TEST

\section{Introduction}

Mouse behavioural models are a cornerstone of the research on genetic determinants of psychiatric disorders in humans. Genetically engineered mice are designed to mimic behavioural and brain abnormalities observed in human subjects. The aim is to pinpoint the genetic determinants of psychiatric conditions (e.g., major depression disorder, schizophrenia, and autism spectrum disorder) and develop effective treatments.

In order to understand the mechanisms driving the onset of psychiatric conditions in both mice and humans, it is vital to link phenotypic (behavioural) and genetic information. Wild-type mice (controls) and genetically modified mice are thus compared on a set of behavioural tests to establish whether the genetic modification of interest (e.g., knock-out or overexpression of a set of genes) causes any meaningful behavioural change.

One of the most frequently employed mouse research tests is the Home Cage Social Interaction test (hereafter HCSI). The HCSI test $[1,2]$ is a standard tool for investigating mouse social behaviour and locomotor activity in a familiar environment. Specifically, this test is designed to record the physical contacts between a pair of mice and the distance they cover over a certain amount of time. The HCSI test is often included in test batteries for the evaluation of several mouse models of psychiatric disorders. Examples include anxiety and depression [3-6], schizophrenia [2,7-9], autism spectrum disorder $[10,11]$ and addiction $[4,12]$.

\section{Nonlinearity in HCSI Data}

Despite their relevance in the investigation of mouse behaviour, there is no established protocol concerning how HCSI data should be examined. HCSI data are usually analysed with linear modelling techniques such as ANOVA or simple linear regression (e.g., the studies mentioned above). However, the HCSI test is often performed over a period of several days. Consequently, the response (dependent) variables of interest (i.e., social interaction and locomotor activity) are affected by the mouse's circadian rhythm (e.g., increased activity during nights). The variables follow a cyclic, nonlinear, 24-hour pattern repeating throughout the test (Figure 1). Therefore, any linear modelling method is most likely inadequate for handling HCSI data. 


\section{GAM FOR THE HCSI TEST}
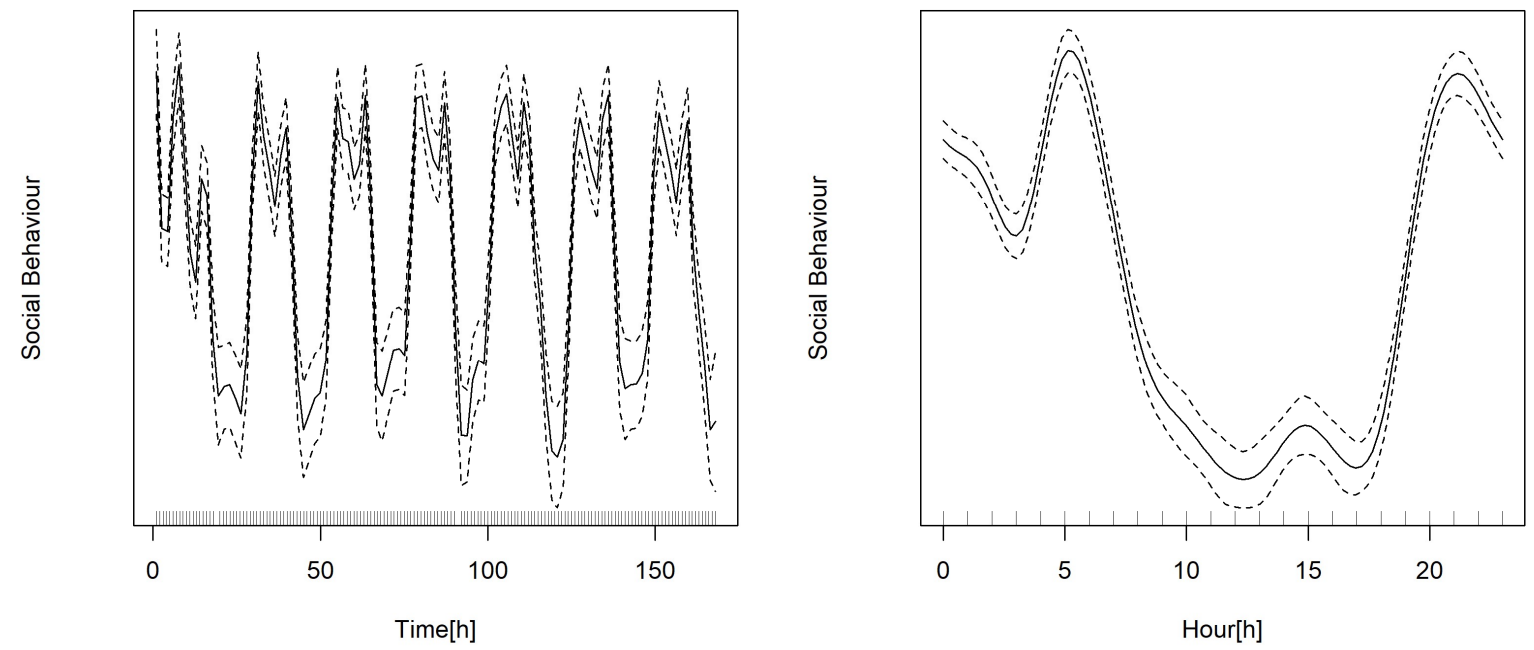

Figure 1. An example of model-estimated trends in the HCSI test (QRFP_1st mouse strain, social behaviour). The left panel illustrates the trend throughout the whole trial (7 days). The right panel shows 24-hour trend that repeatedly occurs over the days. Both trends exhibit a clear nonlinear relationship with the response variable.

Averaging the data (e.g., using only means of the whole trial period) or analysing night data and day data separately are not valid avenues to solve the issue. These analytic strategies may eliminate (averaging) or reduce (separating day and night data) the degree of curvilinearity in the data. Nevertheless, a considerable amount of valuable information about the mouse's phenotypic characteristics would be lost.

A more sophisticated way to approach the problem would be employing polynomial regression [13]. However, polynomial functions are often erratic and, thus, provide quite a poor fit in some places of the function's domain (for more details, see [14] Chapter 4). Furthermore, building an ad-hoc parametric function to analyse a given dataset presupposes a specific statistical model (e.g., an $n$-order polynomial with known $n$ ) developed in previous empirical investigation. To the best of our knowledge, no such model has ever been designed or tested for the HCSI test.

\section{Non-Normality in HCSI Data}

Another issue may emerge from the statistical assumptions regarding the mean-variance relationship in the HCSI variables distribution. Simple linear modelling techniques often assume a normal distribution in the response variable. That means that the variable is thought to have a certain mean $(\mu)$ and a constant variance $\left(\sigma^{2}\right)$. Therefore, $\mu$ and $\sigma^{2}$ are supposed to be independent. Conversely, if $\sigma^{2}$ increases as a function of $\mu$, then it is necessary to switch to a non-Gaussian 
GAM FOR THE HCSI TEST

family distribution (e.g., as in Generalized Linear Modelling). However, the mean-variance relationship is rarely (if ever) checked in HCSI data.

\section{Generalized Additive Modelling}

Without a statistical model that handles HCSI data's intrinsic nonlinearity and individuates the correct mean-variance relationship, it is not possible to produce reliable results. Specifically, violating the assumptions of linearity and homoscedasticity (i.e., constant variance) may lead to an incorrect estimation of the model's parameters such as standard errors and $p$-values. These miscalculations sometimes result in incorrect decisions to reject/retain the null hypothesis and biased effect sizes. Furthermore, without applying the correct model, it is hard to tell when the violation of the above assumptions has little practical consequences. In a nutshell, discriminating between trustworthy and unreliable findings becomes impossible without any well-established model.

Generalized Additive Modelling (GAM) constitutes a suitable solution to both these issues. GAM [14,15] extends linear modelling by incorporating a sum of smooth functions of covariates (i.e., independent variables). These smooth functions allow the relationship between covariates and response variables to be nonlinear, non-monotonic, and, when necessary, cyclic (for a user-friendly introduction to GAM, see [16]). Also, GAM can implement exponential family distributions other than the Gaussian. Finally, GAM does not require the researcher to specify the model's prior structure, like, for example, what function should be implemented (e.g., polynomial, exponential, or a combination of several different functions). For these reasons, GAM is ideal in those circumstances where, due to the lack of models to test, an explorative approach is necessary. ${ }^{1}$

GAM is a useful analytical tool in numerous disciplines such as environmental sciences [17], medicine [18], and, more recently, behavioural sciences [19]. For example, GAM can be implemented to study mean temperature trends in a given city or region over a period of several years ([14], pp. 371-373). Daily mean temperatures follow a 365-day seasonal cyclic pattern, making linear modelling unsuitable for extracting the overall trend throughout several years. By contrast, GAM can easily dissociate the mean temperature trend over the years from the cyclic component (the day of the year) by implementing smooth function for cyclic data. The same approach can be used to model HCSI data.

\footnotetext{
${ }^{1} \mathrm{~A}$ drawback of GAM is that, like any other explorative machine-learning technique, it is computationally much heavier compared to parametric techniques. Nonetheless, this is no longer a major issue nowadays because commercially available computers can run GAM models in a reasonable amount of time.
} 


\section{GAM FOR THE HCSI TEST}

\section{The Present Investigation}

We aim to demonstrate that (a) the methods commonly used to analyse HCSI data may produce inaccurate results, and (b) GAM consistently improves the model's fit and thus yields more reliable results than linear modelling. In order to do so, we examine a publicly available database that includes several statistically independent HCSI trials. We investigate the impact of using nonlinear modelling and non-Gaussian family distributions on the model's fit (e.g., explained deviance) and null hypothesis testing (i.e., rejection/non-rejection of $\mathrm{H} 0$ ).

We focus on the two most common response (dependent) variables of interest of the HCSI test, that is, locomotor activity and social behaviour. Researchers working with genetically engineered mice are primarily concerned about the mouse's genotype's effect on behaviour. Another aspect often catching the researcher's attention is the effect of the mouse's genotype throughout the whole period of the experiment. Therefore, the covariates (independent variables) examined in the present work are mouse genotype, the time since the beginning of the experiment and its interaction with genotype, and the hour of the day in which the response variables are recorded.

\section{Method}

\section{Preliminary Literature Search}

We implemented a systematic search strategy [20] to check if any previous studies employed nonlinear modelling to analyse HCSI data in the last 20 years (2001-2020). By using the following Boolean string (mouse OR mice) AND ("home cage") AND (nonlinear* OR gam), we searched through Complementary Index database. We retrieved 256 records. As of September $30^{\text {th }}$, 2020, none of these studies reported any nonlinear analysis with regard to the HCSI test. No previous study seems to have employed either GAM or any other nonlinear modelling techniques with HCSI data based on this search.

\section{Variables}

Locomotor activity (numeric, response variable, range $[0, \infty)$ ). The mouse's locomotion was given by the total number of recorded pixels that change between time frames (one per second) in one hour.

Social behaviour (numeric, response variable, range [1, 2]). In each of the 1-second frames, either one particle or two particles were recorded. Two particles indicated that the two mice were not in contact. When the two mice were in contact, only one particle was recorded. The mean recorded particles gave the mouse's social behaviour in an hour. We subtracted 1 to this value to 
GAM FOR THE HCSI TEST

obtain a $[0,1]$ range, which was more treatable in the context of non-Gaussian family distributions (for details, see below).

Time (numeric, covariate, range [1, 168]). The number of time points (hours) since the beginning of the experiment. ${ }^{2}$

Hour (numeric, covariate, range [0,23]). The hour of the day at which the response variable was recorded. This variable represented the cyclic component of the GAM models (for details, see below).

Genotype (2-level factor, covariate). The genotype of the pair of mice (Control or Mutant).

Pair (factor, random effect). The pair of mice. Each pair included two mice of the same genotype.

\section{Datasets}

The data were retrieved from the Mouse Phenotype Database (for all the details, see http://www.mouse-phenotype.org/). We examined this database because it is, to the best of our knowledge, the largest publicly available database with regard to the HCSI test. It contains 28 HCSI datasets that include two groups of mice (i.e., controls and mutants). One of these datasets reports some anomalies in the Social behaviour variable (many values $>2$ ) and was excluded. We thus analysed 27 datasets. The total number of mice was 846 (423 pairs). For the complete list of the mouse strains, see the Supplemental Materials.

\section{Models}

We considered three different models to describe the relationship between the variables. Model 1 was the commonly used mixed linear model, while Model 2 and Model 3 were generalized additive models. All the analyses were run with the $m g c v$ R package $[21,22]$.

Model 1. Linear mixed model. This type of model assumes that the response variable $(y)$ is normally distributed with mean $\mu$ and variance $\sigma^{2}$. The predictor variables or covariates (model matrix $X$ ) are multiplied by some coefficients (vector $\beta$ ) and summed to give a linear predictor for $y:$

$$
y \sim \mathcal{N}\left(\mu, \sigma^{2}\right)
$$

\footnotetext{
${ }^{2}$ Time may be modelled as a categorical variable (i.e., 167 degrees of freedom) in a repeatedmeasures ANOVA. We here omit this case because artificially discretizing a continuous variable is often not recommendable. That said, applying a repeated-measures ANOVA on the present data exacerbates the problems observed in Model 1 (i.e., violation of fundamental statistical assumptions and, hence, incorrect $p$-value estimation).
} 
GAM FOR THE HCSI TEST

$$
y=X \beta+Z b+\epsilon,
$$

where $Z$ is a model matrix of random effects, $b \sim \mathcal{N}\left(0, I \sigma_{b}^{2}\right)$ is a vector of random effects, and $\epsilon \sim \mathcal{N}\left(0, I \sigma^{2}\right)$ is the vector of residuals. $b$ and $\epsilon$ are independent. In our case, the covariates were Time, Genotype, and their interaction. Pair was the random variable. The response variable was either Locomotor_activity or Social_Behaviour. Therefore, the formula for Model 1 was:

$$
y=\beta_{0}+\beta_{1} \text { Genotype }+\beta_{2} \text { Time }+\beta_{3} \text { Genotype } * \text { Time }+f_{r}(\text { Pair })+\epsilon,
$$

where $f_{r}$ was a random effect function.

Model 2. Generalized additive model (GAM). This model extended Model 1 by including an additive structure of both a parametric (linear) component and a set of additive smooth functions. The general formula for a GAM model is expressed as follows:

$$
\begin{gathered}
y \sim \operatorname{ExpoFam}(\mu, \ldots), \\
g(\mu)=X \beta+f_{1}\left(x_{1}\right)+f_{2}\left(x_{2}\right)+\cdots f_{p}\left(x_{p},\right),
\end{gathered}
$$

where $x_{i}$ are the covariates, ExpoFam is an Exponential Family distribution, $\mu \equiv E(y), g$ is a link function relating $\mu$ to the addictive formula, and $f_{i}$ are smooth functions of one or more covariates. Like in Model 1, we employed the Gaussian family distribution and an identity link function for the response variable.

The formula for Model 2 is:

$$
\begin{gathered}
y \sim \mathcal{N}\left(\mu, \sigma^{2}\right), \\
y=\beta_{0}+\beta_{1} \text { Genotype }+f_{1}(\text { Time })+f_{2}(\text { Genotype, Time })+f_{3}(\text { Hour })+f_{r}(\text { Pair })+\epsilon,
\end{gathered}
$$
where $f_{1}$ and $f_{2}$ were smooth functions with cubic spline basis, while $f_{3}$ was a smooth a that employs a cyclic cubic spline basis. This latter term was essential to take into account the cyclic pattern observed in the response variables due to the mouse circadian rhythms.

Model 3. This model modified Model 2 by implementing non-Gaussian family distributions. Model 1 and Model 2 arbitrarily assumed that the response variable $y$ was normally distributed with constant variance (homoscedasticity). However, this assumption may be untenable in HCSI data. The locomotor activity variable may present a larger dispersion as its values increase. Furthermore, the social behaviour variable is both left- and right-censored (domain $[0,1]$ ), which is most likely incompatible with the assumption of normality.

Model 3 thus implemented two non-Gaussian family distributions, that is, the Tweedie and the Quasi-Binomial for the locomotor activity and social behaviour, respectively. The Tweedie 
GAM FOR THE HCSI TEST

distribution $[23,24]^{3}$ has mean $\mu \equiv E(y)$, a positive dispersion parameter $\sigma^{2}$, and $\operatorname{Var}(y)=\sigma^{2} \mu^{p}$, where $1<p<2$ is the Tweedie power parameter that must be estimated. We applied a log link function (the standard with the Tweedie distribution). The Quasi-Binomial distribution [25] is defined by the mean $\mu \equiv E(y)$, (assuming that $0 \leq y \leq 1$ ), the number of recorded trials $n,{ }^{4}$ and the dispersion parameter $\varphi$. A logit link was applied.

The formula of Model 3 was thus expressed as follows:

$$
y=\beta_{0}+\beta_{1} \text { Genotype }+f_{1}(\text { Time })+f_{2}(\text { Genotype, Time })+f_{3}(\text { Hour })+f_{r s}(\text { Time, } \text { Pair })+\epsilon,
$$

where we assumed that the response variable follows a Tweedie distribution $y \sim T w\left(\mu, \sigma^{2}, p\right)$ and a Quasi-binomial distribution $y \sim Q b(\mu, n, \varphi)$, in the locomotor activity model and the social behaviour model, respectively. We also replaced simple random effects $f_{r}$ (Pair))with random smooth curves $f_{r s}$ (Time, Pair). These smooth curves represented the random deviation from the mean trajectory of each pair of mice throughout the whole experiment.

\footnotetext{
${ }^{3}$ The Quasi-Poisson and the negative binomial family distributions may be valid alternatives. ${ }^{4}$ This parameter is set on 60 (i.e., the number of minutes per hour, as reported in the Mouse Phenotype Database).
} 


\section{GAM FOR THE HCSI TEST}

\section{Results}

\section{Fit Comparison between Model 1 and Model 2}

Model 2 largely outperformed Model 1 in all the 27 trials in both the response variables.

Model 2 explained a significantly greater amount of deviance (all $p$-values $<2.2 \times 10^{-16}$; Chi-squared test). The results are depicted in Figure 2 (for all the details, see Table S1). ${ }^{5}$
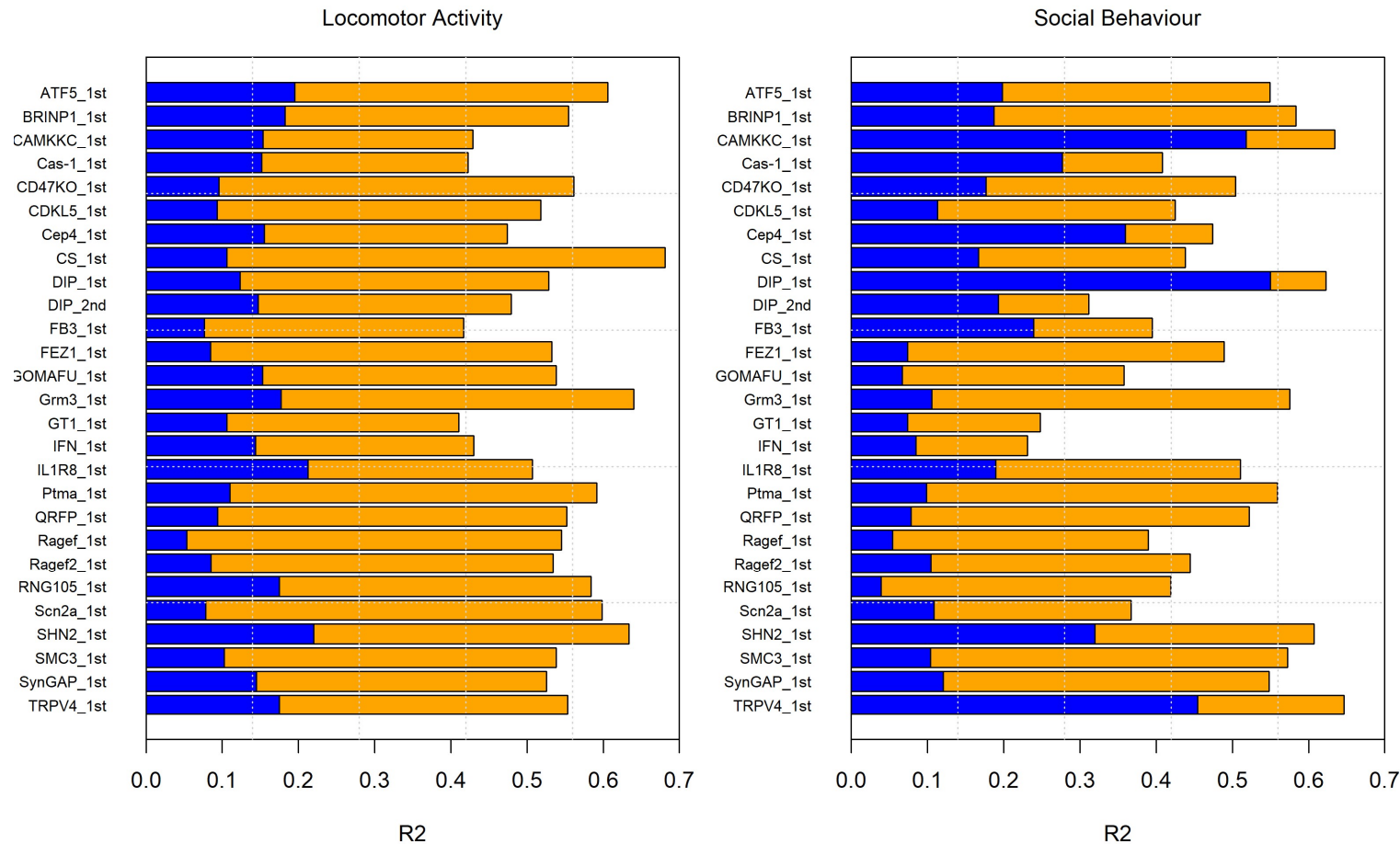

Figure 2. Summary of the explained deviance $\left(R^{2}\right)$ in all the 27 mouse strains for locomotor activity (left panel) and social behaviour (right panel). The blue bars represent Model 1's explained deviance, while the orange bars are the difference between Model 2's and Model 1's explained deviance. Model 2 proves to be systematically superior to Model 1.

The inclusion of the cyclic smooth function and the other non-cyclic smooth functions - which allowed for nonlinearity and non-monotonicity - compellingly improved the HCSI models' fit. Therefore, as expected, Model 1's assumption of linearity was substantially invalid.

\section{Reliability Comparison between Model 1 and Model 3}

The inspection of the residuals and fitted values highlighted that the Gaussian family distribution was unsuitable to the data. Some fitted values were negative, which should be impossible for the response variables (all data points $\geq 0$ ). Furthermore, while the power

${ }^{5}$ The explained variances indexes of Model 3 can be retrieved from the R codes. They are not reported in Figure 2 because explained deviance in Model 3 is expressed in a different metric due to the link functions (log and logit for locomotor activity and social behaviour, respectively). 


\section{GAM FOR THE HCSI TEST}

parameter $p$ was expected to be 0 under the Gaussian assumption, the actual $p$ was positive in nearly all the cases. This result constituted convincing evidence of overdispersion (i.e., $\sigma^{2}$ increased as a function of $\mu$ ). Thus, both Model 1 and, to a lesser degree, Model 2 were unsatisfactory (for all the diagnostics plots, see the Supplemental Materials).

As described above, the Tweedie and the Quasi-Binomial exponential family distributions were used in Model 3 for locomotor activity and social behaviour, respectively. As expected, the deviance residuals' distribution is systematically much closer to normality compared to both Model 1 and Model 2. An example is shown in Figure 3 (for all the diagnostic plots, see the Supplemental Materials). Overall, the results produced by Model 3 were thus more reliable than the other two sets of models.
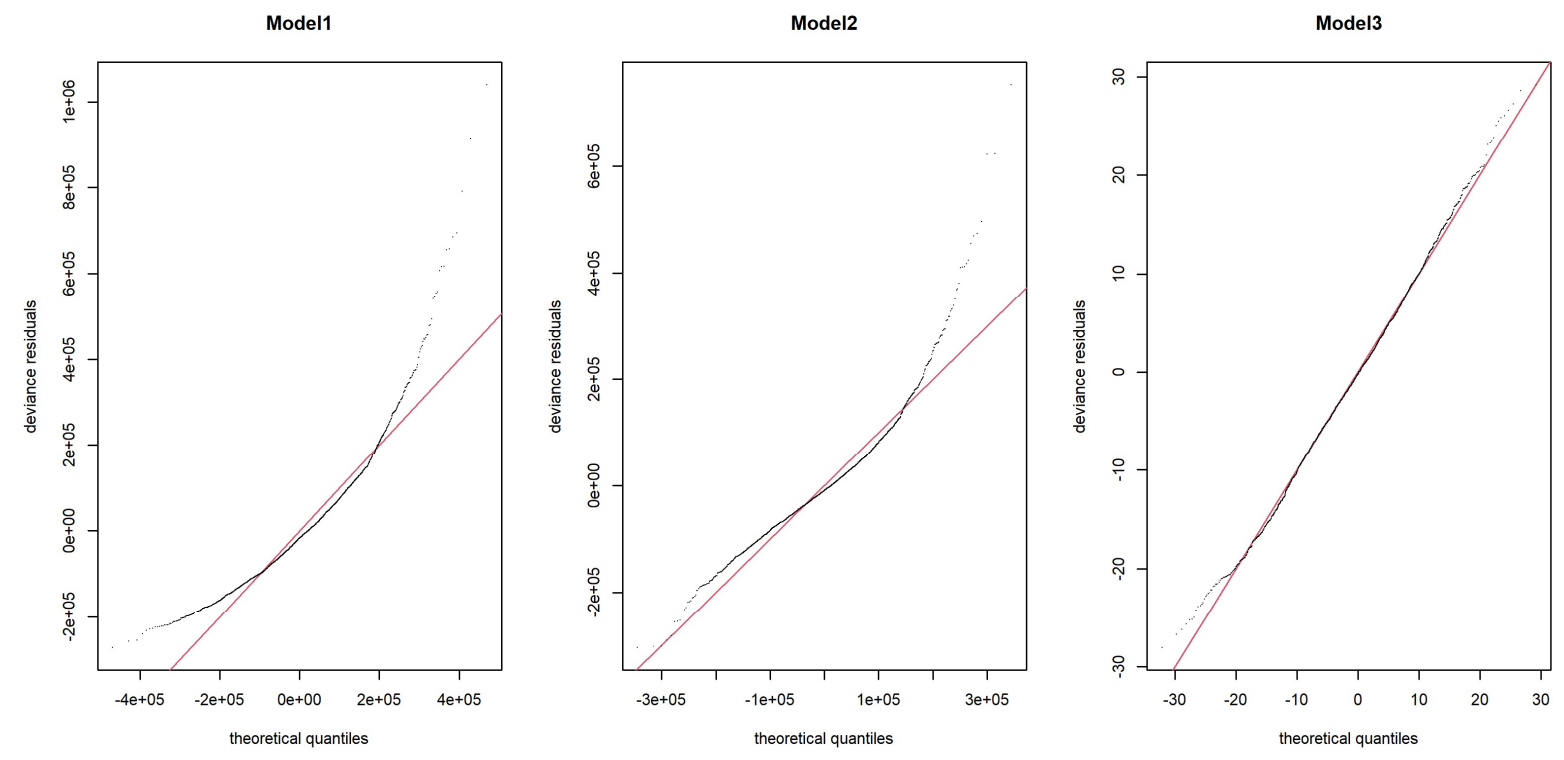

Figure 3. Example of QQ-plots of the residuals of the three models (locomotor activity in DIP_1st strain). The distribution of the residuals in Model 1 (left panel) indicates a problem with the assumption of Gaussian distribution (the dots, that is, the residuals often far from their theoretical values represented by the straight red line). The inclusion of a critical covariate (i.e., the cyclic component) mitigates the problem in Model 2, but the residuals' distribution remains unsatisfactory (centre panel). By contrast, Model 3 (right panel; residuals in log scale) presents no significant issue.

Finally, Model 1 and Model 3 reached different conclusions about the Genotype effect and Time-Genotype interaction. In fact, Model 1 appeared to be more prone to yield statistically significant results ( $p$-value $<.05$, two-tailed) with regard to the Genotype effect, while the opposite pattern occurred with the Time-Genotype interaction (Table 1; for all the details, see Table S2 and Table S3). 
GAM FOR THE HCSI TEST

Table 1

Number of significant and non-significant p-values in Model 1 and Model 3

\begin{tabular}{llccc}
\hline \multicolumn{1}{c}{ Model } & \multicolumn{1}{c}{ Effect } & $\boldsymbol{p}$-values $<\mathbf{. 0 5}$ & $\boldsymbol{p}$-values $\geq \mathbf{. 0 5}$ & $\begin{array}{c}\text { Equal } \\
\text { Decisions }\end{array}$ \\
\hline Model 1 - LA & Genotype & 15 & 12 & \multirow{2}{*}{$21(6)$} \\
\hline Model 3 - LA & Genotype & 9 & 18 & \multirow{2}{*}{$22(5)$} \\
\hline Model 1 - LA & Time-Genotype interaction & 10 & 17 & \multirow{2}{*}{$22(5)$} \\
\hline Model 3 - LA & Time-Genotype interaction & 13 & 14 & \multirow{2}{*}{$16(11)$} \\
\hline Model 3 - SB & Genotype & 8 & 19 & 24 \\
\hline Model 1 - GB & Timotype & 3 & 19 & 14 \\
\hline
\end{tabular}

${ }^{a}$ The number of times Model 1 and Model 3 reach the same conclusion (rejection or failure to reject $\mathrm{H} 0$ ). The number of inconsistent decisions is in brackets. $\mathrm{LA}=$ locomotor activity; $\mathrm{SB}=$ social behaviour. Comparisons are based on 27 datasets.

\section{Discussion}

The present article has focused on how to analyse HCSI data (locomotor activity and social behaviour). As seen, virtually no previous study has adopted any nonlinear technique to model mouse performance in the HCSI test, although these data systematically exhibit cyclic patterns. We have employed a nonlinear modelling technique (i.e., GAM) to address this issue.

GAM has systematically proved to be far more accurate and predictive than linear modelling. Model 2 outperforms Model 1 on all the 27 datasets in the two response variables (i.e., greater $R^{2}$ values and smaller sums of residuals). The inclusion of the cyclic component, the smoothing function of the variable Hour, accounts for most of the additional - i.e., from Model 1 to Model 2 - explained deviance (see $F$-values in Table S3). It is worth noting that the cyclic component's role is crucial, even when the researcher has no specific interest in phenotypic characteristics related to mouse circadian cycles. The omission of an important (the most important in the present case) covariate in a model often disrupts the distribution of the model's residuals, which affects the calculation of standard errors and $p$-values. Thus, the inclusion of the cyclic component is necessary to correctly estimate the magnitude and statistical significance of the group effect of interest (e.g., mutants vs. controls, Table S4).

Finally, Model 3 extends Model 2 by including random smooth curves for each pair of mice and, most importantly, employing non-Gaussian exponential family distributions (i.e., Tweedie and Quasi-Binomial). This latter adjustment allows the models to meet those statistical assumptions (e.g., correct mean-variance relationship) that Model 1 and, to a lesser extent, Model 2 fail to satisfy. The results yielded by Model 3 are, therefore, the most trustworthy. 


\section{GAM FOR THE HCSI TEST}

The incorrect assumption of linearity and the use of the Gaussian family distribution in Model 1 bring practical consequences. The inaccurate estimation of the Genotype parametric coefficients ( $\beta$ and standard error) in Model 1 leads to the miscalculation of the $p$-value. In 11 cases out of 54, Model 1 finds a significant Genotype effect, whereas Model 3 fails to reject the null hypothesis. Model 1 thus exhibits a consistent tendency towards Type I error (about 20\% of the total cases). In the same vein, the estimated magnitude of the Genotype effect (Cohen's $d$ ) in Model 1 is sometimes quite different compared to Model 3 (for all the details, see Tables S4).

Interestingly, the opposite pattern of results occurs in the Time-Genotype interaction. While Model 1 finds this interaction to be statistically significant in one-third of the cases (18 out of 54), Model 3 reports 26 significant interactions. These latter results are all but surprising. The interaction between Time and Genotype is arbitrarily assumed to be linear in Model 1 (e.g., strong Genotype effect at the beginning of the experiment progressively fading away). However, genetic modifications (e.g., knockout genes) often affect mouse behaviour through time in a nonlinear fashion. For example, genetically modified mice may show increased locomotor activity or social behaviour only in night hours (cyclic pattern). Any linear model would struggle at spotting such an interaction. Conversely, GAM can easily pick up both linear and nonlinear interactions. Model 3 can thus provide useful information about mouse phenotypic traits that otherwise may remain undetected in Model 1. Figure 4 depicts a simple example (for all the other trend plots, see the Supplemental Materials).
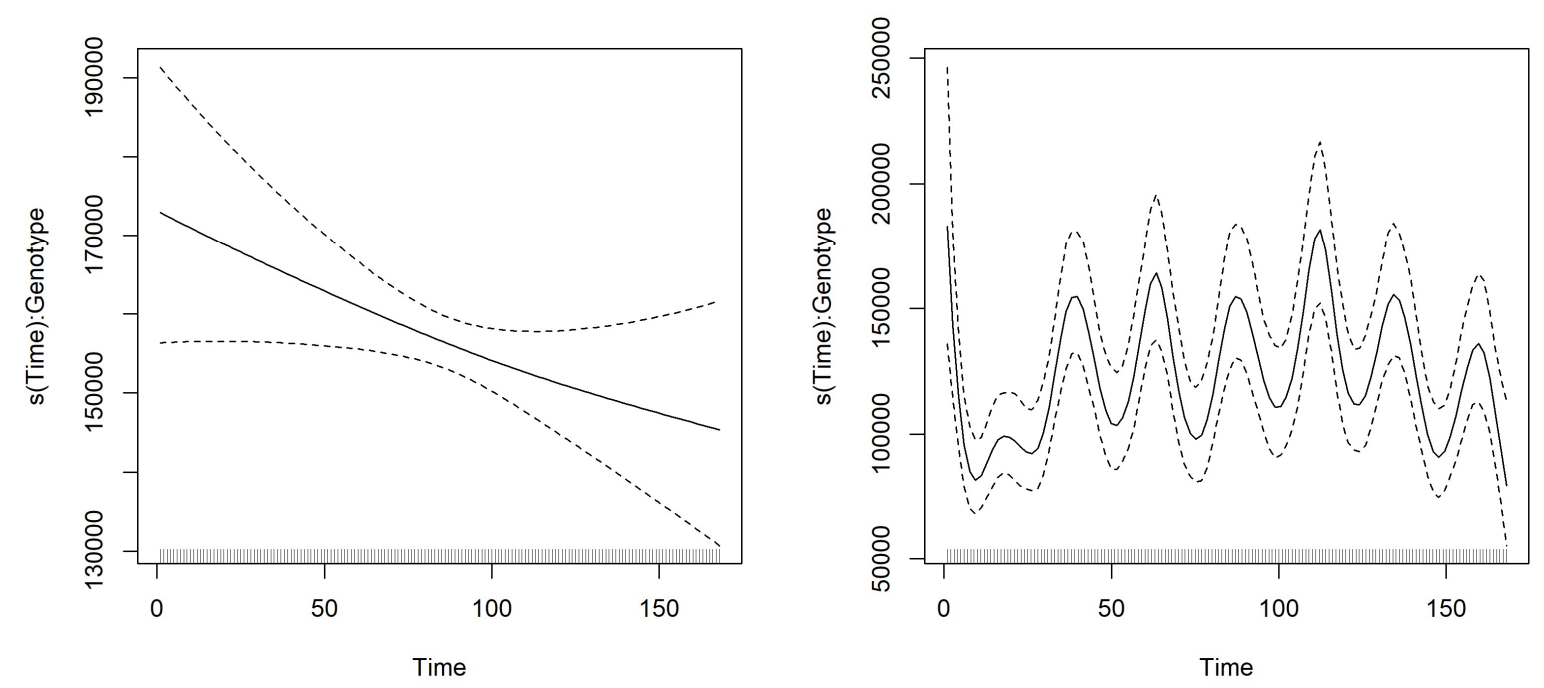

Figure 4. Example of the trend (continuous line) of the estimated differences in locomotor activity ( $y$-axis) between the mutants and controls throughout the 168 hours ( $x$-axis) of the experiment. The dashed lines represent 1 standard error. The left panel shows a linear trend (GOMAFU_1st strain) 


\section{GAM FOR THE HCSI TEST}

that both Model 1 and Model 3 detect (both $p$ s <.001). The right panel (SynGAP_1st strain) reveals an evident cyclic trend that Model 1 fails to recognize $(p=.080)$, whereas Model 3 correctly finds it statistically significant $(p<.001)$.

In brief, Model 1 - which represents the standard way HCSI data are analysed - often yields some unreliable results. Thus, the current reported findings regarding mouse performance in the HCSI test are, to a non-negligible degree, unreliable as well. More broadly, we expect a significant portion of previous HCSI experiments to have come to incorrect conclusions in terms of null hypothesis significance testing and, more generally, estimation of model parameters.

As already mentioned, the HCSI test is often included in behavioural tasks assessing mouse models of numerous psychiatric disorders. Given its prominent role in the field, HCSI data should be analysed with a statistical model that satisfies fundamental statistical assumptions (e.g., correct mean-variance relationship) and explain a fair amount of observed variability (deviance). We think that Model 3 meets both conditions. We thus recommend that researchers employ Model 3.

Obviously, GAM does not necessarily have to be the only suitable method. Other models may be implemented as long as they are equally powerful in terms of explained deviance and precise when it comes to meeting statistical assumptions.

Finally, mathematical accuracy aside, a robust statistical model for analysing HCSI data would be desirable to boost the overall replicability and trustworthiness of experimental outcomes [26-28]. In the absence of such a model, researchers would inevitably analyse their data with different and most likely suboptimal/incorrect models. Such flexibility in data analysis may lead to unreproducible and inconsistent findings, or even questionable research practices such as $p$-hacking [29] and HARKing [30]. Consequently, we firmly believe that the overall quality of mouse behaviour assessment would benefit from adopting standard statistical models that meet all the fundamental statistical assumptions.

\section{Conclusions}

The present work shows that the assumption of linearity, the omission of a cyclic component, and the use of the Gaussian family distribution often lead to unreliable results in the HCSI test. In other words, the statistical model customarily assumed in the analysis of HCSI data is incorrect. Therefore, commonly employed linear methods are inadequate for analysing HCSI data, which are nonlinear and cyclic due to mouse circadian rhythms. Conversely, GAM systematically produces far better results in terms of both reliability and explained deviance. Thus, we recommend 


\section{GAM FOR THE HCSI TEST}

employing GAM, or any other equivalently powerful analytic tool, to improve the quality and trustworthiness of HCSI results. 
GAM FOR THE HCSI TEST

\section{References}

1. Crawley JN. What's wrong with my mouse? Behavioral phenotyping of transgenic and knockout mice. 2nd ed. John Wiley \& Sons Inc; 2007. doi:10.1002/9780470119051

2. Powell CM, Miyakawa T. Schizophrenia-relevant behavioral testing in rodent models: A uniquely human disorder? Biol Psychiatry. 2006;59: 1198-1207. doi:10.1016/j.biopsych.2006.05.008

3. Maeta K, Hattori S, Ikutomo J, Edamatsu H, Bilasy SE, Miyakawa T, et al. Comprehensive behavioral analysis of mice deficient in Rapgef2 and Rapgef6, a subfamily of guanine nucleotide exchange factors for Rap small GTPases possessing the Ras/Rap-associating domain. Mol Brain. 2018;11. doi:10.1186/s13041-018-0370-y

4. Nakamura H, Yamashita N, Kimura A, Kimura Y, Hirano H, Makihara H, et al. Comprehensive behavioral study and proteomic analyses of CRMP2-deficient mice. Genes to Cells. 2016;21: 1059-1079. doi:10.1111/gtc.12403

5. Niemann S, Kanki H, Fukui Y, Takao K, Fukaya M, Hynynen MN, et al. Genetic ablation of NMDA receptor subunit NR3B in mouse reveals motoneuronal and nonmotoneuronal phenotypes. Eur J Neurosci. 2007;26: 1407-1420. doi:10.1111/j.1460-9568.2007.05774.x

6. Tatsukawa T, Raveau M, Ogiwara I, Hattori S, Miyamoto H, Mazaki E, et al. Scn2a haploinsufficient mice display a spectrum of phenotypes affecting anxiety, sociability, memory flexibility and ampakine CX516 rescues their hyperactivity. Mol Autism. 2019;15. doi:10.1186/s13229-019-0265-5

7. Miyakawa T, Leiter LM, Gerber DJ, Gainetdinov RR, Sotnikova TD, Zeng H, et al. Conditional calcineurin knockout mice exhibit multiple abnormal behaviors related to schizophrenia. Proc Natl Acad Sci U S A. 2003;100: 8987-8992.

doi:10.1073/pnas. 1432926100

8. Tremolizzo L, Doueiri MS, Dong E, Grayson DR, Davis J, Pinna G, et al. Valproate corrects the schizophrenia-like epigenetic behavioral modifications induced by methionine in mice. Biol Psychiatry. 2005;57: 500-509. doi:10.1016/j.biopsych.2004.11.046

9. Wiedholz LM, Owens WA, Horton RE, Feyder M, Karlsson RM, Hefner K, et al. Mice lacking the AMPA GluR1 receptor exhibit striatal hyperdopaminergia and "schizophreniarelated" behaviors. Mol Psychiatry. 2008;13: 631-640. doi:10.1038/sj.mp.4002056

10. Nakajima R, Takao K, Hattori S, Shoji H, Komiyama NH, Grant SGN, et al. Comprehensive 


\section{GAM FOR THE HCSI TEST}

behavioral analysis of heterozygous Syngap1 knockout mice. Neuropsychopharmacol Reports. 2019;39: 223-237. doi:10.1002/npr2.12073

11. Paemka L, Mahajan VB, Skeie JM, Sowers LP, Ehaideb SN, Gonzalez-Alegre P, et al. PRICKLE1 interaction with SYNAPSIN I reveals a role in autism spectrum disorders. PLoS One. 2013;8: e80737. doi:10.1371/journal.pone.0080737

12. Morishima Y, Miyakawa T, Furuyashiki T, Tanaka Y, Mizuma H, Nakanishi S. Enhanced cocaine responsiveness and impared motor coordination in metabotropic glutamate receptor subtype 2 knockout mice. Proc Natl Acad Sci U S A. 2005;102: 4170-4175. doi:10.1073/pnas.0500914102

13. Barbur VA, Montgomery DC, Peck EA. Introduction to Linear Regression Analysis. Stat. 1994;43: 339. doi:10.2307/2348362

14. Wood SN. Generalized additive models: An introduction with R. 2nd ed. London: NY: CRC Press; 2017. doi:10.1201/9781315370279

15. Hastie T, Tibshirani R. Generalized additive models. Stat Sci. 1986;1: 297-318. doi: $10.1214 /$ ss/1177013604

16. Clark M. Generalized Additive Models. 2019. Available: https://mclark.github.io/generalized-additive-models/

17. Yee TW, Mitchell ND. Generalized additive models in plant ecology. J Veg Sci. 1991;2: 587-602. doi:10.2307/3236170

18. Hastie T, Tibshirani R. Generalized additive models for medical research. Stat Methods Med Res. 1995;4: 187-196. doi:10.1177/096228029500400302

19. Vaci N, Gula B, Bilalić M. Is age really cruel to experts? Compensatory effects of activity. Psychol Aging. 2015;30: 740-754. doi:10.1037/pag0000056

20. Appelbaum M, Cooper H, Kline RB, Mayo-Wilson E, Nezu AM, Rao SM. Journal article reporting standards for quantitative research in psychology: The APA publications and Communications Board task force report. Am Psychol. 2018;73: 3-25. doi:10.1037/amp0000191

21. Wood SN. Package 'mgcv'. 2019. doi:10.1201/9781315370279

22. Team RC. R: A Language and Environment for Statistical Computing. Vienna, Austria. 2019. 


\section{GAM FOR THE HCSI TEST}

23. Jørgensen B. Exponential dispersion models. J R Stat Soc Ser B. 1987;49: 127-162. doi:10.1111/j.2517-6161.1987.tb01685.x

24. Tweedie MCK. An index which distinguishes between some important exponential families. Applications and New Directions-Proceedings of the Indian Statistical Institute Golden Jubilee International Conference. 1984. pp. 579-604.

25. McCullagh P, Nelder JA. Generalized Linear Models. London: NY: Chapman \& Hall; 1989.

26. Kafkafi N, Agassi J, Chesler EJ, Crabbe JC, Crusio WE, Eilam D, et al. Reproducibility and replicability of rodent phenotyping in preclinical studies. Neurosci Biobehav Rev. 2018;87: 218-232. doi:10.1016/j.neubiorev.2018.01.003

27. Open Science Collaboration. Estimating the reproducibility of psychological science. Science (80- ). 2015;6251: aac4716. doi:10.1126/science.aac4716

28. Miyakawa T. No raw data, no science: Another possible source of the reproducibility crisis. Mol Brain. 2020;13: 24. doi:10.1186/s13041-020-0552-2

29. Simmons JP, Nelson LD, Simonsohn U. False-positive psychology: Undisclosed flexibility in data collection and analysis allows presenting anything as significant. Psychol Sci. 2011;22: 1359-1366. doi:10.1177/0956797611417632

30. Kerr NL. HARKing: Hypothesizing after the results are known. Personal Soc Psychol Rev. 1998;2: 196-217. doi:10.1207/s15327957pspr0203_4 